\title{
Spontaneous spinal epidural haematoma with spontaneous resolution
}

\author{
L.A. Brawn, U.E.G. Bergval and G.A.B. Davies-Jones \\ Department of Neurology, Royal Hallamshire Hospital, Sheffield S10 2JF, UK.
}

\begin{abstract}
Summary: Spontaneous spinal epidural haematoma is of unknown aetiology, but it has been associated with hypertension. Treatment is surgical decompression but three cases of spontaneous recovery have been reported. We report the fourth such recovery in a 68 year old hypertensive woman.
\end{abstract}

\section{Introduction}

Spinal epidural haematoma is a rare condition, with only about 200 cases reported in the literature. Approximately half of all cases are caused by identifiable abnormalities such as vascular malformations, trauma, epidural anaesthesia, coagulation disorders and anticoagulant treatment. The remainder are considered to be spontaneous but may be associated with systemic hypertension.

Early surgery is the treatment of choice (McQuarrie, 1978). Spontaneous recovery from spinal epidural haematomas has been reported only rarely (Harik $\boldsymbol{e t}$ al., 1971; Hernandex et al., 1982; Priest, 1935). We describe a further case of spontaneous spinal epidural haematoma with spontaneous recovery.

\section{Case report}

A 68 year old woman developed a sudden onset of severe low back pain while bending forwards. Pain radiated down the left leg initially followed by pain down the right leg, associated with bilateral parasthesiae. After 27 hours the left leg suddenly became paralysed followed by paralysis of the right leg a few minutes later.

There was no history of recent trauma or anticoagulation. She was hypertensive taking oxprenolol $160 \mathrm{mg} /$ day.

On examination, she was in pain and had a blood pressure of $180 / 110 \mathrm{mmHg}$. She had a complete areflexic flaccid paralysis of the legs and absent plantar responses. There was a dense, complete sensory level to all modalities at T10. The bladder was not palpable,

Correspondence: G.A.B. Davies-Jones, M.D., F.R.C.P., Department of Neurology, Royal Hallamshire Hospital, Sheffield S10 2JF, UK.

Accepted: 8 April 1986 and she had a lax anal sphincter.

A lumbar myelogram was performed 29 hours after the onset of pain and showed multiple stenoses at the lumbar discs from L1-L4 with displacement of the roots of the cauda equina. The examination was technically difficult and the thoracic region could not be seen adequately. When reassessed 34 hours after the onset, the power in her legs had improved and pinprick sensation had returned above the knees.

A cervical myelogram performed at 40 hours showed an extradural expanding lesion with dorsal compression of the subarachnoid space over segments T3-T5 and traces of contrast below this level (Figure 1a). These appearances with further clinical improvement suggest a diagnosis of extradural haematoma and conservative management was continued.

Over the next 3 days, muscle power returned. Repeat myelography 14 days later showed less extradural compression allowing contrast to run through easily (Figure 1b). These findings were compatible with a resolving extradural haematoma. The cerebrospinal fluid showed 32 white cells $/ \mathrm{mm}^{3}(50 \%$ polymorphs, $50 \%$ lymphocytes) and protein $2 \mathrm{~g} / \mathrm{l}$. (The fluid obtained initially was normal.)

After 2 months she was fully recovered with normal power, sensation, bladder and bowel function and normal anal sphincter tone.

Further investigations showed normal clotting studies, platelet count, erythrocyte sedimentation rate and serum globulin. The patient declined to have a spinal CT scan or spinal angiography.

\section{Discussion}

The clinical picture was typical of spinal extradural haematoma, with sudden pain, a rapid onset of 


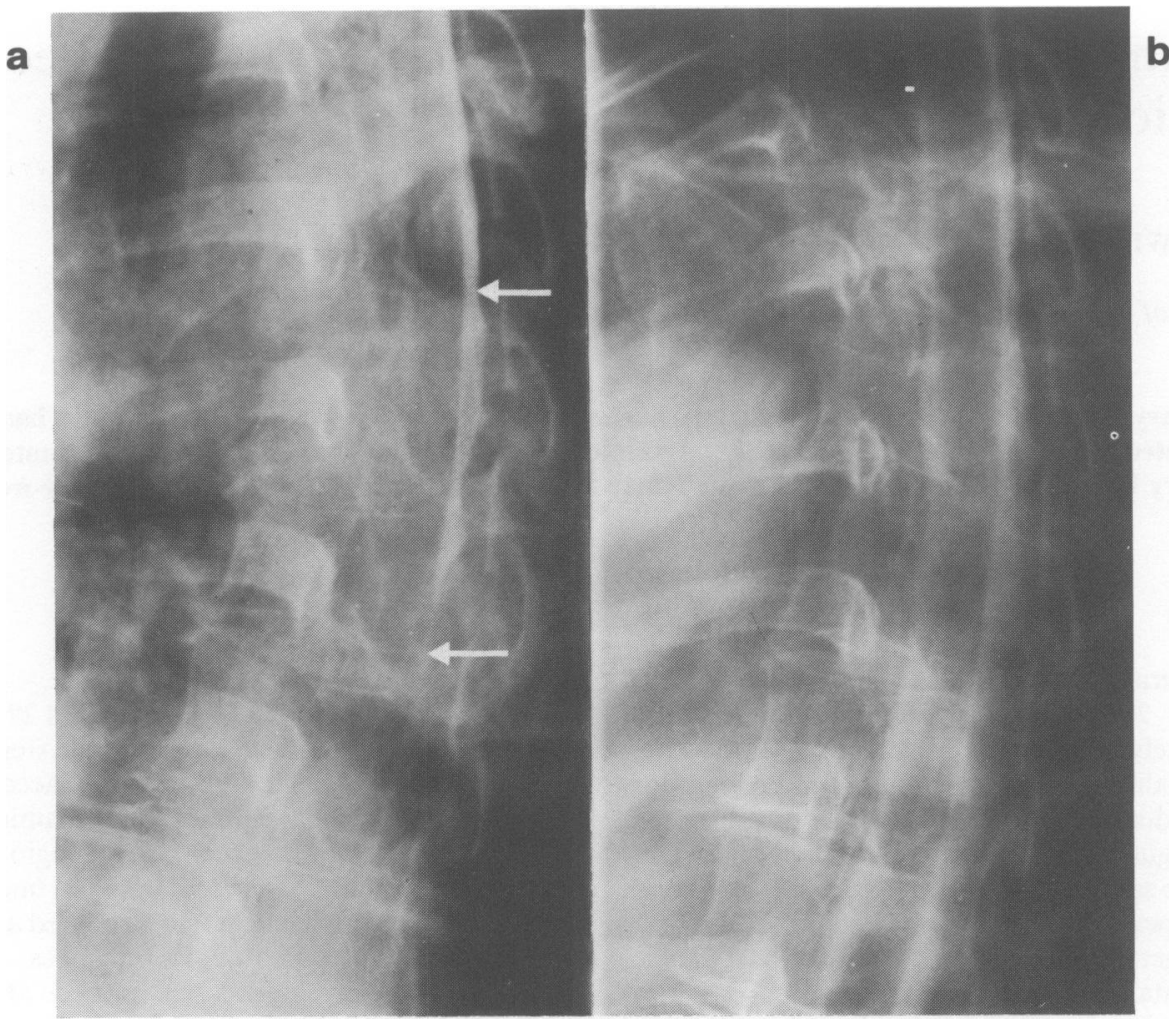

Figure 1 (a) Cervical myelogram - dorsal compression over segments T3-T5 between the two arrows.

(b) Considerable improvement.

areflexic paralysis and characteristic sensory loss. The diagnosis was confirmed by myelography which inititally demonstrated an almost complete block from a dorsal compressive lesion, and was later shown to resolve. These appearances were compatible with a diagnosis of extradural haematoma, but could not be confirmed with a CT scan at the time. The complete clinical recovery excluded other causes of extradural compression. In this case, there was no identifiable cause for the haematoma, so called 'spontaneous', although there was an association with hypertension.

The pathogenesis of spontaneous spinal epidural haematoma is obscure (Beatty \& Winston, 1984). Haemorrhage from the epidural venous plexus has been suggested. This is thought to be due to transmission of raised intra-abdominal or intrathoracic pressure from apparently mild trauma or straining. But cases have been described occurring during sleep. An arterial source and microscopic subperiosteal vascular malformations have also been suggested. The latter cannot be excluded in this case since laminectomy was not performed, and angiography was declined.

The lesion may occur anywhere along the spinal canal and usually extends over 2-3 segments. Whatever the site, surgery is generally mandatory. A delay of greater than 36 hours before decompresson is associated with a poor recovery (McQuarrie, 1978). In this case, the rapid clinical improvement at 7 hours after the onset of paralysis favoured a conservative approach.

Spontaneous recovery from extradural haematomas has been described rarely. One case occurred in a haemophiliac (Priest, 1935) and another in a patient on anticoagulants (Harik et al., 1971). In both of these cases surgery was felt to be contraindicated. A third such recovery occurred spontaneously, in a hypertensive, diabetic patient who had a relapsing and remitting clinical course, with the longest episode of paralysis lasting 120 minutes (Hernandez et al., 1982). We believe that our case is the fourth example of spontaneous recovery of an extradural haematoma. 


\section{References}

BEATTY, R.M. \& WINSTON, K.R. (1984). Spontaneous cervical epidural haematoma. A consideration of aetiology. Journal of Neurosurgery, 61, 143.

HERIK, S.I., RAICHLE, M.E. \& REIS, D.J. (1971). Spontaneously remitting epidural haematoma in a patient on anticoagulants. New England Journal of Medicine, 284, 1355.

HERNANDEZ, D., VINCULAR, F. \& FEASBY, T.E. (1982).
Recurrent paraplegia with total recovery from spontaneous spinal epidural haematoma. Annals of Neurology, 11, 623 ,

MCQUARRIE, L.G. (1978). Recovery from paraplegia caused by spontaneous spinal epidural haematoma. Neurology, 28, 224.

PRIEST, W.M. (1935). Epidural haemorrhage due to haemophilia. Lancet, ii, 1289. 